\title{
Drug resistance and plasmid profile of bacterial isolates from automated teller machine keypads in Benin city
}

\author{
F. I. Akinnibosun* and J. A. Adetitun
}

Department of Microbiology, Faculty of Life Sciences, University of Benin, P.M.B 1154, Benin City, Edo State, Nigeria

Received: 18 September 2018

Revised: 25 November 2018

Accepted: 03 December 2018

DOI: 10.3329/bjsir.v54i3.42675

\begin{abstract}
The purpose of this study was to investigate the drug resistance and plasmid profile of bacterial isolates from automated teller machine (ATM) keypads in Benin City, Nigeria. Samples were collected from the keypads of ATMs situated in banking premises and open access areas using stratified random sampling method at 5 different locations. Isolated bacteria were characterized by extensive phenotypic and biochemical identification procedures. Antibiotic susceptibility pattern and plasmid profile of the isolates were carried out using standard methods. The bacteria isolated were: Klebsiella sp., Pseudomonas aeruginosa, Proteus vulgaris, Escherichia coli, Micrococcus luteus, Bacillus subtilis, Staphylococcus aureus, Corynebacteria sp., Staphylococcus epidermidis and Streptococcus pyogenes. Each isolate exhibited marked resistance to the antibiotics, with multiple antibiotic resistance (MAR) index $>0.2$. Escherichia coli showed the highest resistance (MAR index, 1) while Micrococcus luteus exhibited the least resistance (MAR index, 0.5) to the antibiotics used. Plasmid profile of the isolates revealed the presence of plasmids in the isolates, with a reduction in antibiotic resistance after plasmid curing in some isolates. This indicated plasmid-mediated resistance among majority of the isolates. From the results obtained, it is pertinent therefore, to adopt proper measures aimed at preventing outbreak of diseases associated with these multi-drug resistant bacteria isolated from ATM keypads.
\end{abstract}

Keywords: Antibiotics; disease; Automated Teller Machines; Multidrug resistance; Plasmid extraction

\section{Introduction}

The contamination of diverse objects by probable pathogenic microorganisms is of public health significance, as contaminated materials can be possible font of spread of such pathogens. Bacteria have been substantiated to be spread from person to person by way of contact with fomites. Some of these bacteria are opportunistic pathogens to humans and animals, and cause diseases in individuals (Short et al., 2007). Generally, members of the public seem not to be aware that microorganisms dwell on many common objects in the open, workplace and likewise in their dwelling. As a result of the ongoing development and digitization, as well as the increasing population, people do not have enough time to use traditional banking systems and have embraced new developments in electronic banking, such as automated teller machines. Today, the extended use of electronic technologies is considered a source of bacterial contamination (Saroja et al., 2013).

Microorganisms coexist with the majority of life on earth, including human beings, and in several instances are indispensable for the continued existence of their host. Therefore it is not always beneficial to eradicate them by sterilizing our environs. Merely areas where the adhesion of microorganisms is undesirable should be sanitized or decontaminated. Such antimicrobial surface amendments are required in the therapeutic sphere and in material discipline (Bieser et al., 2011). Some of these microorganisms are hard to obliterate and usually persist for a prolonged period on floors and different types of non-porous surfaces. The spread of resistant strains to our houses and public places where people carry out their daily activities is on the increase. Therefore, there is need to encourage consistent 
decontamination of regularly-used items as part of an efficient approach to thwart the proliferation of multi-drug resistant pathogens and the incidence of ensuing infections (Maillard, 2005).

The imminent concern is that the amount of bacterial strains which acquire resistance against disinfectants and notably antibiotics is on the increase faster than anticipated. Owing to the recurrent threat to human life, extermination or regulating the development of pathogenic microorganisms specifically bacteria, viruses and fungi on non-living surfaces remain to be a chief interest all over the world (Hamouda and Baker, 2000). Antimicrobial agents have been employed for several years to overcome pathogenic microorganisms in an ample range of functions (in hospitals, the home and industrial premises). Nevertheless, using them for a protracted period has resulted in the development of defiant microorganisms (Maillard, 2002). The amount of antibiotic resistant bacteria has escalated in contemporary years due to the abuse of antibiotics and biocides which in numerous cases have resulted in the emergence of cross resistance. Consequently, novel, benign and effective biocides are constantly required to overcome complications related with microorganism's modification and the emergence of resistant strains (Hamouda and Baker, 2000).

Automated Teller Machine, an automated telecommunication device that aid it users or financial institution clients to execute financial dealings without the need for a cashier, bank clerk or bank teller. Running as a data station interacting via a host processor which network all other such technology operated by a bank across a wide area grid, it makes money withdrawal and other services accessible to the account-holder further expedient (Sharma and Anand, 2002). It standard usage encompass slipping the required card into a recipient slot and following onscreen directives, by striking the metallic keypads to enter confidential codes and instructions; hence charging the machine as to the sort of service one needs (Sharma and Anand, 2002). The device is liable to be soiled with innumerable microorganisms due to their immeasurable dermal contact by several users. Pathogens proliferate amongst people with direct or indirect contact with hands or on inanimate paraphernalia (Mathai et al., 2010).

Bank automated teller machines (ATMs) are mainly localized in metropolitan centres, merchandize areas, and around the infirmary. Quite a lot of people whose socioeconomic levels and aseptic status are rather different from each other utilize ATMs daily. The point of contact is the customer's hands to the exterior of keypad and/or display of this piece of equipment. Point has been raised that contact with soiled computer keyboards might serve as a means for contaminating user hands with probable pathogens, resulting in cross-contamination of operators (Steffen et al., 2008).

In view of these, it is imperative to examine the antibiotic susceptibility and plasmid profile of bacterial isolates on ATM keypads used by different people under everyday conditions and in various institutions or organizations and to investigate the presence of multidrug resistant bacteria.

\section{Materials and methods}

\section{Study area}

Benin City, the seat of government of Edo State is situated in the southern part of Nigeria. Its location is geographically defined by latitudes $6^{\circ} 11^{\prime}$ and $6^{\circ} 29^{\prime} \mathrm{N}$ and longitude $5^{\circ} 47^{\prime} \mathrm{E}$. The metropolitan area traverse four local government areas: Egor, Oredo, Ovia North-East and Ikpoba-Okha. The capital has continued to be a key commercial focal point associating the western, eastern, northern and southern parts of Nigeria. The overall landscape of Benin City can be said to be a low and gentle slope from about $105 \mathrm{~m}$ above shoreline in the North-East to $55 \mathrm{~m}$ in the South-West area. Temperature is typically elevated with a yearly mean of $28{ }^{\circ} \mathrm{C}$. The municipality faces a yearly rainfall beyond $2000 \mathrm{~mm}$, with average relative humidity exceeding $80 \%$.

\section{Sample collection}

Samples were collected from the ATM keypads of machines situated in the banking premises and those in open access areas, shared by an array of different users or open to the general public. Samples were collected using stratified random sampling method at five different locations. The command keys of ATMs were swabbed on five separate days; between the hours of 06:00 am to 08:00 am and 14:00 $\mathrm{pm}$ and 16:00 pm. The swabs were instantaneously immersed into marked tubes of nutrient concentrate immediately on arrival at the laboratory. Control arrangements were fashioned by dipping pristine swabs in labelled broth tubes.

\section{Isolation and characterisation of bacteria}

All samples were cultured by the pour-plate method on Nutrient agar and incubated at $28{ }^{\circ} \mathrm{C}$ for 24 hours. Colonies were sub-cultured recurrently on immaculate media until 
pure cultures were obtained. The isolates were then typified using their colonial, cellular morphology, and biochemical characteristics, according to Holt et al. (1994) and Breed et al. (1975).

\section{Antibiotic susceptibility test}

This was done according to Bauer et al. (1966) and as described by National Committee for Clinical Laboratory Standards (NCCLS) (2000 and 2005). The concentration of the ten standard antibiotics used were: Penicillins

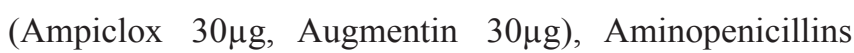

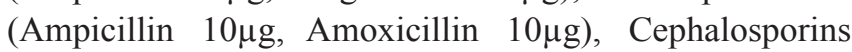
(Cefuroxime 30 $\mu \mathrm{g}$, Ceftriaxone $30 \mu \mathrm{g}$ ), Aminoglycosides

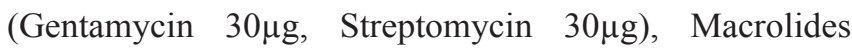

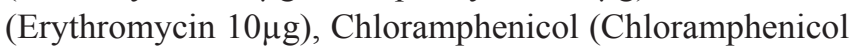
$10 \mu \mathrm{g}$ ), Fluoroquinolones (Ciprofloxacin $10 \mu \mathrm{g}$, Ofloxacin $5 \mu \mathrm{g}$, Pefloxacin $10 \mu \mathrm{g}$ ) and Trimethoprim/Sulfonamides (Septrin $25 \mu \mathrm{g}$ ). Standardized culture of every single isolate was made by picking two distinct colonies using a sterile wire-loop and emulsifying them in $3 \mathrm{ml}$ of saline in a sample bottle and then incubated at $28{ }^{\circ} \mathrm{C}$ overnight. The standardized overnight culture of each isolate was used to flood the surface of Mueller-Hinton agar plates. The surplus was poured off and the plates were left to dry. The standard antibiotic disc was then sterilely placed at rational equidistance on the inoculated Muller-Hinton agar plate with sterilized forceps and then lightly pushed down onto the agar surface for even contact. The plates were incubated aerobically for $18-24 \mathrm{~h}$ at $28{ }^{\circ} \mathrm{C}$. The zones of inhibition around each disk was measured and recorded in millimetres. The results were also recorded according to CLSI (2013), as either susceptible (S), intermediate (moderate resistant) (I) or resistant (R) to the antimicrobial agents used.

\section{Multiple antibiotic resistance}

Multiple Antibiotic Resistance (MAR) Index is a functional delineation tool in bacterial source tracking and risks investigation by evaluating resistance profile of bacteria versus antibiotics used. MAR was calculated as reported by Zhou et al. (1990)

$$
M A R=\frac{a}{b}
$$

Where; $\mathrm{a}=$ number of antibiotics to which isolate is resistant; $\mathrm{b}=$ total number of antibiotics tested.

\section{Plasmid profile}

A measured volume of $2-3 \mathrm{ml}$ of freshly grown culture was centrifuged, and the pellet was re-suspended in $1 \mathrm{ml}$ of solution comprising $0.04 \mathrm{M}$ Tris-acetate, $(\mathrm{pH} 8.0)$ and 2 mM EDTA. Also, $2 \mathrm{ml}$ lyses buffer, $0.05 \mathrm{M}$ Tris, $3 \%$ SDS, $\mathrm{pH} 12.5$, regulated with 2 molar $\mathrm{NaOH}$ was mixed gently; the suspension was incubated at $68^{\circ} \mathrm{C}$ for 45 mins. A $6 \mathrm{ml}$ of phenol/chloroform (1:1) was supplemented to the hot samples and mixed lightly to achieve emulsification. Phases were separated by centrifugation at $10000 \mathrm{rev} / \mathrm{s}$ for 20 mins, and the upper aqueous phase was displaced carefully to pristine microfuge tube containing $400 \mu \mathrm{l}$ of chloroform. This was mixed and centrifuged again for partition of phases. The aqueous phase was recovered and used promptly for agarose gel electrophoresis (Zhou et al., 1990).

Plasmid Curing and Re-evaluation of antibiotic susceptibility

Curing of plasmids was performed in consensus to the technique described by Akortha and Filgona (2009). A $5 \mathrm{ml}$ aliquot of overnight suspension culture of bacteria was sub-cultured into tubes holding $5 \mathrm{ml}$ of nutrient broth complemented with $0.1 \mathrm{mg} / \mathrm{ml}$ acridine orange and then incubated at $37^{\circ} \mathrm{C}$ for $48 \mathrm{~h}$. The bacterial cultures were then plated out on Mueller-Hinton agar and used in the re-examination of the antibiotic susceptibility pattern as expressed above.

\section{Results and discussion}

\section{Characterization and prevalence of bacterial isolates}

Ten different bacteria were found associated with ATM keypads in Benin metropolis (Table I). The bacterial isolates were: Klebsiella sp., Pseudomonas aeruginosa, Proteus vulgaris, Escherichia coli, Micrococcus luteus, Bacillus subtilis, Staphylococcus aureus, Corynebacteria sp., Staphylococcus epidermidis and Streptococcus pyogenes. Table II showed the prevalence of the bacterial isolates. Bacillus subtilis (10.15\%), Micrococcus luteus (3.75\%), Escherichia coli (12.58 \%), Pseudomonas aeruginosa (7.28 $\%)$, Streptococcus pyogenes (11.58 \%), Corynebacterium sp. $(8.60 \%)$, Klebsiella sp. (8.16\%), Staphylococcus aureus (13.46\%), Proteus vulgaris (8.83), and Staphylococcus epidermidis (19.42\%). 
Table I. Cultural, morphological and biochemical characteristics

\begin{tabular}{|c|c|c|c|c|c|c|c|c|c|c|}
\hline & 1 & 2 & 3 & 4 & 5 & 6 & 7 & 8 & 9 & 10 \\
\hline \multicolumn{11}{|l|}{ Cultural } \\
\hline Shape & Circular & Circular & Circular & Irregular & Circular & Circular & Circular & Irregular & Circular & Circular \\
\hline \multirow[t]{2}{*}{ Elevation } & Convex & Convex & Low & Flat & Convex & Convex & Convex & Flat & Convex & Convex \\
\hline & & & convex & & & & & & & \\
\hline Margin & Entire & Entire & Entire & Undulate & Entire & Entire & Entire & Serrated & Entire & Entire \\
\hline Wetness/dryness & Wet & Wet & Wet & Dry & Wet & Wet & Wet & Wet & Wet & Wet \\
\hline Transparency & Opaque & Opaque & Opaque & Opaque & Opaque & Opaque & Opaque & Opaque & Opaque & Opaque \\
\hline \multirow[t]{2}{*}{ Colour } & Yellow & Cream & Green & Cream & Yellow & Dull & Whitish & Cream & Cream- & Cream \\
\hline & & & & & & \multicolumn{2}{|l|}{ cream } & & \multicolumn{2}{|l|}{ white } \\
\hline Size & Medium & Medium & Medium & Large & Small & Medium & Small & Large & Medium & Small \\
\hline \multicolumn{11}{|l|}{ Morphological } \\
\hline Gram staining & \multicolumn{10}{|l|}{+} \\
\hline Cell type & Cocci & Rod & Rod & Rod & Cocci & Rod & Cocci & Rod & Cocci & Rod \\
\hline Cell arrangement & Single & Single & Single & Chain & Cluster & Single & Chain & Single & Cluster & Single \\
\hline \multicolumn{11}{|l|}{ Biochemical } \\
\hline Catalase & + & + & + & + & + & + & - & + & + & + \\
\hline Oxidase & - & - & + & - & - & - & - & - & - & - \\
\hline Coagulase & - & - & - & - & + & - & - & - & - & - \\
\hline Urease & + & + & - & + & + & + & + & + & + & - \\
\hline Indole & - & - & - & - & - & - & - & + & - & + \\
\hline Citrate & + & + & + & + & + & + & + & + & + & - \\
\hline \multicolumn{11}{|l|}{ Sugar } \\
\hline \multicolumn{11}{|l|}{ fermentation } \\
\hline Glucose & A & $\mathrm{AG}$ & A & A & A & A & A & A & A & $\mathrm{AG}$ \\
\hline Lactose & - & + & - & - & - & - & - & - & - & + \\
\hline \multicolumn{11}{|l|}{ Possible isolates } \\
\hline & 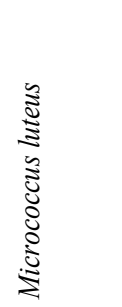 & 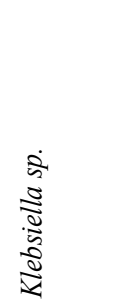 & 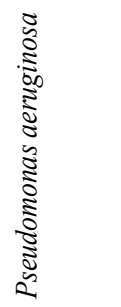 & 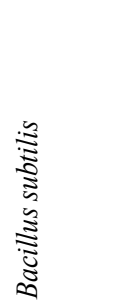 & 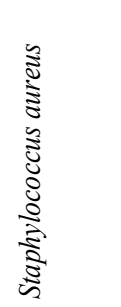 & 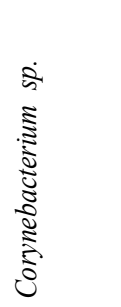 & 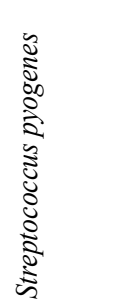 & 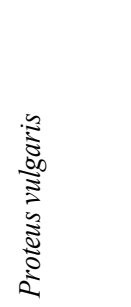 & 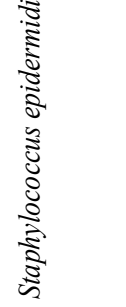 & 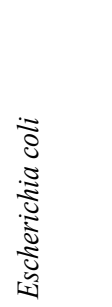 \\
\hline
\end{tabular}


Table II. Prevalence of bacterial isolates

\begin{tabular}{lc}
\hline Isolates & Prevalence (\%) \\
\hline Escherichia coli & 12.58 \\
Pseudomonas aeruginosa & 7.28 \\
Streptococcus pyogenes & 11.58 \\
Corynebacterium sp. & 8.60 \\
Klebsiella sp. & 8.16 \\
Staphylococcus aureus & 13.46 \\
Proteus vulgaris & 8.83 \\
Micrococcus luteus & 3.75 \\
Bacillus subtilis & 10.15 \\
Staphylococcus epidermidis & 19.42 \\
\hline
\end{tabular}

Table III. Antibiotic susceptibility of isolates before curing

\begin{tabular}{|c|c|c|c|c|c|c|c|c|c|c|c|c|c|}
\hline $\begin{array}{l}\text { Isolates } \\
\text { (Gram } \\
\text { Negative) }\end{array}$ & 离 & 巴ֶ & $\sum_{i}^{\infty}$ & 爻 & $\sum^{\circlearrowright}$ & 昰 & $\sum_{\circlearrowleft}$ & $\frac{\mathrm{I}}{\mathrm{T}}$ & 宏 & $\sim$ & 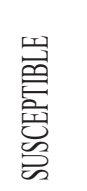 & 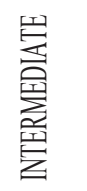 & 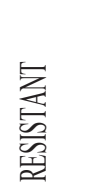 \\
\hline Klebsiella sp. & $\mathrm{R}$ & $\mathrm{R}$ & $\mathrm{R}$ & I & $\mathrm{R}$ & $\mathrm{R}$ & $\mathrm{R}$ & $\mathrm{R}$ & $\mathrm{R}$ & $\mathrm{R}$ & $0 \%$ & $10 \%$ & $90 \%$ \\
\hline $\begin{array}{l}\text { Pseudomonas } \\
\text { aeruginosa }\end{array}$ & $\mathrm{R}$ & $\mathrm{R}$ & $\mathrm{R}$ & I & $\mathrm{R}$ & $\mathrm{R}$ & $\mathrm{R}$ & $\mathrm{R}$ & $\mathrm{R}$ & $\mathrm{R}$ & $0 \%$ & $10 \%$ & $90 \%$ \\
\hline Proteus vulgaris & I & $\mathrm{R}$ & $\mathrm{R}$ & I & $\mathrm{R}$ & $\mathrm{R}$ & S & $\mathrm{R}$ & $\mathrm{R}$ & I & $10 \%$ & $30 \%$ & $60 \%$ \\
\hline Escherichia coli & $\mathrm{R}$ & $\mathrm{R}$ & $\mathrm{R}$ & $\mathrm{R}$ & $\mathrm{R}$ & $\mathrm{R}$ & $\mathrm{R}$ & $\mathrm{R}$ & $\mathrm{R}$ & $\mathrm{R}$ & $0 \%$ & $0 \%$ & $100 \%$ \\
\hline $\begin{array}{l}\text { Isolates } \\
\text { (Gram Positive) }\end{array}$ & 䆓 & $\sum_{\circlearrowright}$ & 齐 & $N$ & $\sum_{i}^{U}$ & $\simeq$ & 气ّ̂ & $\sim$ & 离 & 피 & & & \\
\hline $\begin{array}{l}\text { Micrococcus } \\
\text { luteus }\end{array}$ & $\mathrm{R}$ & $\mathrm{S}$ & $\mathrm{R}$ & $\mathrm{R}$ & $\mathrm{R}$ & $\mathrm{R}$ & $\mathrm{S}$ & I & I & I & $20 \%$ & $30 \%$ & $50 \%$ \\
\hline Bacillus subtilis & $\mathrm{R}$ & $\mathrm{S}$ & $\mathrm{R}$ & $\mathrm{R}$ & $\mathrm{R}$ & $\mathrm{R}$ & I & I & $\mathrm{R}$ & $\mathrm{S}$ & $20 \%$ & $20 \%$ & $60 \%$ \\
\hline $\begin{array}{l}\text { Staphylococcus } \\
\text { aureus }\end{array}$ & $\mathrm{R}$ & $\mathrm{S}$ & $\mathrm{R}$ & $\mathrm{R}$ & $\mathrm{R}$ & $\mathrm{R}$ & I & $\mathrm{R}$ & $\mathrm{R}$ & I & $20 \%$ & $10 \%$ & $70 \%$ \\
\hline $\begin{array}{l}\text { Corynebacteriu } \\
m \text { sp. }\end{array}$ & $\mathrm{R}$ & $\mathrm{S}$ & $\mathrm{R}$ & $\mathrm{R}$ & $\mathrm{R}$ & $\mathrm{R}$ & I & $\mathrm{R}$ & $\mathrm{R}$ & I & $10 \%$ & $20 \%$ & $70 \%$ \\
\hline $\begin{array}{l}\text { Streptococcus } \\
\text { pyogenes }\end{array}$ & $\mathrm{R}$ & $\mathrm{S}$ & $\mathrm{R}$ & $\mathrm{R}$ & $\mathrm{R}$ & $\mathrm{R}$ & $\mathrm{R}$ & $\mathrm{R}$ & $\mathrm{R}$ & I & $10 \%$ & $10 \%$ & $80 \%$ \\
\hline $\begin{array}{l}\text { Staphylococcus } \\
\text { epidermidis }\end{array}$ & $\mathrm{R}$ & S & $\mathrm{R}$ & $\mathrm{R}$ & $\mathrm{R}$ & $\mathrm{R}$ & I & $\mathrm{R}$ & $\mathrm{R}$ & I & $10 \%$ & $20 \%$ & $70 \%$ \\
\hline
\end{tabular}

$\mathrm{SXT}=$ Septrin $($ Sulfamethoxazole + Trimethoprim $), \mathrm{CH}=$ Chloramphenicol, $\mathrm{AMP}=$ Ampicillin, $\mathrm{CPX}=\mathrm{Ciprofloxacin}$, $\mathrm{AMC}=$ Amoxicillin, $\mathrm{AUG}=$ Augmentin, $\mathrm{GM}=$ Gentamycin, $\mathrm{PEF}=$ Pefloxacin, $\mathrm{OFX}=$ Ofloxacin, $\mathrm{S}=$ Streptomycin, $\mathrm{APX}=$ Ampiclox, $\mathrm{Z}=$ Zinacef (Cefuroxime), $\mathrm{R}=$ Rocephin $($ Ceftriaxone $), \mathrm{E}=$ Erythromycin 


\section{Antimicrobial susceptibility of isolates}

All the isolates showed marked resistance to Chloramphenicol, Ampicillin, Amoxicillin, Augmentin, Pefloxacin and Ofloxacin (Table 3). Escherichia coli, Klebsiella sp., Pseudomonas aeruginosa and Proteus vulgaris showed $100 \%, 90 \%, 90 \%$ and $60 \%$ resistance respectively, to all the antibiotics. Proteus vulgaris showed 10\% susceptibility to Gentamycin. Streptococcus pyogenes showed $80 \%$ resistance to gentamycin while Micrococcus luteus showed $50 \%$ resistance to gentamycin. All isolates showed intermediate resistance to Erythromycin except Bacillus subtilis which displayed marked susceptibility (10\%).

\section{Multidrug resistance profile of isolates}

Each isolate was subjected to ten (10) antibiotics and all isolates showed marked resistance to at least five antibiotics. Escherichia coli showed the highest resistance to all ten
$(100 \%)$ antibiotics followed by Klebsiella sp. and Pseudomonas aeruginosa with resistance to nine of all ten $(90 \%)$ antibiotics. It can be observed from Table 4, that the Gram-negative isolates showed more multidrug resistance than the Gram-positives. Both the Gram-negatives and Gram-positive isolates all showed marked resistance to Pefloxacin while all isolates excluding Proteus vulgaris and Micrococcus luteus were also resistant to Septrin. All the isolates had an MAR (Multi Antibiotic Resistance) Index > 0.2 (Table 4).

\section{Plasmid Profile of Bacterial Isolates}

The plasmid profile of the bacterial isolates is depicted in Fig. 1. Plasmid-mediated resistance was observed in some of the isolates (Escherichia coli, Klebsiella sp., Proteus vulgaris), due to decrease in antibiotic resistance from loss of plasmids after curing as seen in Table V.

Table IV. Multidrug Resistance (MDR) Profile of Isolates

\begin{tabular}{|c|c|c|c|}
\hline Isolates & Resistance pattern of isolates & $\begin{array}{c}\text { Total number of } \\
\text { Antibiotics }(\mathrm{n}=10)\end{array}$ & $\begin{array}{l}\text { MAR (Multi Antibiotic } \\
\text { Resistance) Index }\end{array}$ \\
\hline Klebsiella sp. & 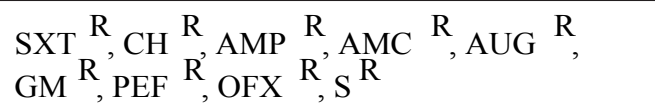 & 9 & 0.9 \\
\hline $\begin{array}{l}\text { Pseudomonas } \\
\text { aeruginosa }\end{array}$ & 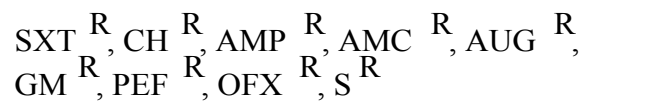 & 9 & 0.9 \\
\hline Proteus vulgaris & 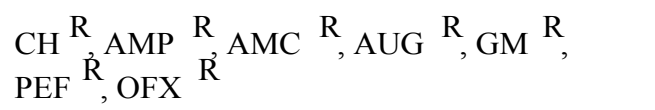 & 7 & 0.7 \\
\hline Escherichia coli & 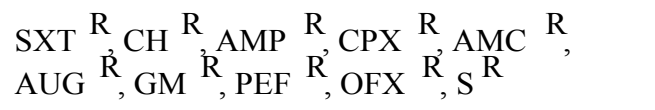 & 10 & 1 \\
\hline Micrococcus luteus & $\mathrm{PEF}^{\mathrm{R}}$, APX ${ }^{\mathrm{R}}, \mathrm{Z}^{\mathrm{R}}$, AMC $^{\mathrm{R}}, \mathrm{R}^{\mathrm{R}}$ & 5 & 0.5 \\
\hline Bacillus subtilis & PEF $^{\mathrm{R}}$, APX ${ }^{\mathrm{R}}, \mathrm{Z}^{\mathrm{R}}$, AMC ${ }^{\mathrm{R}}, \mathrm{R}^{\mathrm{R}}$, SXT ${ }^{\mathrm{R}}$ & 6 & 0.6 \\
\hline $\begin{array}{l}\text { Staphylococcus } \\
\text { aureus }\end{array}$ & PEF ${ }^{\mathrm{R}}$, APX ${ }^{\mathrm{R}}, \mathrm{Z}^{\mathrm{R}}$, AMC ${ }^{\mathrm{R}}, \mathrm{R}^{\mathrm{R}}, \mathrm{S}^{\mathrm{R}}, \mathrm{SXT}^{\mathrm{R}}$ & 7 & 0.7 \\
\hline Corynebacterium sp. & $\mathrm{PEF}^{\mathrm{R}}$, APX ${ }^{\mathrm{R}}, \mathrm{Z}^{\mathrm{R}}$, AMC ${ }^{\mathrm{R}}, \mathrm{R}^{\mathrm{R}}, \mathrm{S}^{\mathrm{R}}, \mathrm{SXT}{ }^{\mathrm{R}}$ & 7 & 0.7 \\
\hline $\begin{array}{l}\text { Streptococcus } \\
\text { pyogenes }\end{array}$ & $\underset{\text { SXT }}{\operatorname{PEF}}{ }_{\mathrm{R}}^{\mathrm{R}}$ APX ${ }^{\mathrm{R}}, \mathrm{Z}^{\mathrm{R}}$, AMC ${ }^{\mathrm{R}}, \mathrm{R}^{\mathrm{R}}, \mathrm{CPX}{ }^{\mathrm{R}}, \mathrm{S}^{\mathrm{R}}$, & 8 & 0.8 \\
\hline $\begin{array}{l}\text { Staphylococcus } \\
\text { epidermidis }\end{array}$ & $\mathrm{PEF}^{\mathrm{R}}$, APX ${ }^{\mathrm{R}}, \mathrm{Z}^{\mathrm{R}}$, AMC ${ }^{\mathrm{R}}, \mathrm{R}^{\mathrm{R}}, \mathrm{S}^{\mathrm{R}}, \mathrm{SXT}{ }^{\mathrm{R}}$ & 7 & 0.7 \\
\hline
\end{tabular}

$\mathrm{SXT}=$ Septrin $($ Sulfamethoxazole + Trimethoprim $), \mathrm{CH}=$ Chloramphenicol, $\mathrm{AMP}=$ Ampicillin, $\mathrm{CPX}=$ Ciprofloxacin, $\mathrm{AMC}=\mathrm{Amoxicillin}$, $\mathrm{AUG}=$ Augmentin, $\mathrm{GM}=$ Gentamycin, $\mathrm{PEF}=$ Pefloxacin, OFX = Ofloxacin, $\mathrm{S}=$ Streptomycin, $\mathrm{APX}=\mathrm{Ampiclox}, \mathrm{Z}=\mathrm{Zinacef}$ (Cefuroxime), $\mathrm{R}=$ Rocephin (Ceftriaxone), $\mathrm{E}=$ Erythromycin 
Table V. Antibiotic susceptibility of isolates after curing

\begin{tabular}{|c|c|c|c|c|c|c|c|c|c|c|c|c|c|}
\hline $\begin{array}{l}\text { Isolates } \\
\text { (Gram Negative) }\end{array}$ & 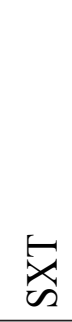 & $\underset{\Xi}{\Xi}$ & $\sum_{i}$ & 己 & $\sum_{i}^{U}$ & \& & $\sum_{\circlearrowleft}$ & $\frac{I}{|c|}$ & 肴 & is & 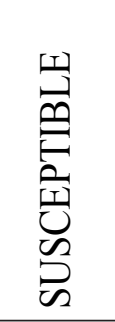 & 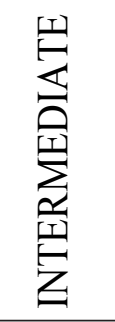 & 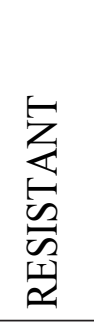 \\
\hline Klebsiella sp. & $\mathrm{R}$ & I & $\mathrm{R}$ & I & $\mathrm{R}$ & $\mathrm{R}$ & $\mathrm{R}$ & $\mathrm{R}$ & $\mathrm{R}$ & $\mathrm{I}$ & $0 \%$ & $30 \%$ & $70 \%$ \\
\hline $\begin{array}{l}\text { Pseudomonas } \\
\text { aeruginosa }\end{array}$ & $\mathrm{R}$ & $\mathrm{R}$ & $\mathrm{R}$ & I & $\mathrm{R}$ & $\mathrm{R}$ & $\mathrm{S}$ & $\mathrm{R}$ & $\mathrm{R}$ & $\mathrm{R}$ & $10 \%$ & $10 \%$ & $80 \%$ \\
\hline Proteus vulgaris & I & $\mathrm{S}$ & $\mathrm{R}$ & I & $\mathrm{R}$ & $\mathrm{R}$ & $\mathrm{S}$ & $\mathrm{R}$ & $\mathrm{R}$ & I & $20 \%$ & $30 \%$ & $50 \%$ \\
\hline Escherichia coli & $\mathrm{R}$ & $\mathrm{S}$ & $\mathrm{R}$ & $\mathrm{R}$ & $\mathrm{R}$ & $\mathrm{R}$ & $\mathrm{R}$ & $\mathrm{R}$ & $\mathrm{R}$ & I & $10 \%$ & $10 \%$ & $80 \%$ \\
\hline $\begin{array}{l}\text { Isolates } \\
\text { (Gram Positive) }\end{array}$ & 㭊 & $\sum_{0}$ & 宏 & $N$ & $\sum_{i}^{U}$ & $\simeq$ & 爻 & $\infty$ & $\begin{array}{l}\sqrt[x]{x} \\
\text { 品 }\end{array}$ & س & & & \\
\hline $\begin{array}{l}\text { Micrococcus } \\
\text { luteus }\end{array}$ & $\mathrm{R}$ & $\mathrm{S}$ & $\mathrm{R}$ & $\mathrm{R}$ & $\mathrm{R}$ & $\mathrm{R}$ & $\mathrm{S}$ & I & $\mathrm{R}$ & I & $20 \%$ & $20 \%$ & $60 \%$ \\
\hline Bacillus subtilis & $\mathrm{R}$ & $\mathrm{S}$ & $\mathrm{S}$ & $\mathrm{R}$ & $\mathrm{R}$ & $\mathrm{R}$ & I & I & $\mathrm{R}$ & $\mathrm{S}$ & $30 \%$ & $20 \%$ & $50 \%$ \\
\hline $\begin{array}{l}\text { Staphylococcus } \\
\text { aureus }\end{array}$ & $\mathrm{R}$ & $\mathrm{S}$ & $\mathrm{R}$ & $\mathrm{R}$ & $\mathrm{R}$ & $\mathrm{R}$ & I & I & $\mathrm{R}$ & I & $20 \%$ & $20 \%$ & $60 \%$ \\
\hline $\begin{array}{l}\text { Corynebacterium } \\
\text { sp. }\end{array}$ & $\mathrm{R}$ & $\mathrm{S}$ & $\mathrm{R}$ & $\mathrm{R}$ & $\mathrm{R}$ & $\mathrm{R}$ & $\mathrm{R}$ & $\mathrm{R}$ & $\mathrm{R}$ & I & $10 \%$ & $10 \%$ & $80 \%$ \\
\hline $\begin{array}{l}\text { Streptococcus } \\
\text { pyogenes }\end{array}$ & $\mathrm{R}$ & $\mathrm{S}$ & $\mathrm{R}$ & $\mathrm{R}$ & $\mathrm{R}$ & $\mathrm{R}$ & $\mathrm{R}$ & $\mathrm{R}$ & $\mathrm{R}$ & $\mathrm{R}$ & $10 \%$ & $0 \%$ & $90 \%$ \\
\hline $\begin{array}{l}\text { Staphylococcus } \\
\text { epidermidis }\end{array}$ & $\mathrm{R}$ & $\mathrm{S}$ & $\mathrm{R}$ & $\mathrm{R}$ & $\mathrm{R}$ & $\mathrm{R}$ & I & S & $\mathrm{R}$ & I & $20 \%$ & $20 \%$ & $60 \%$ \\
\hline
\end{tabular}

$\mathrm{SXT}=$ Septrin $($ Sulfamethoxazole + Trimethoprim $), \mathrm{CH}=$ Chloramphenicol, $\mathrm{AMP}=$ Ampicillin, $\mathrm{CPX}=\mathrm{Ciprofloxacin}, \mathrm{AMC}=$ Amoxicillin, $\mathrm{AUG}=$ Augmentin, $\mathrm{GM}=$ Gentamycin, $\mathrm{PEF}=$ Pefloxacin, $\mathrm{OFX}=$ Ofloxacin, $\mathrm{S}=\mathrm{Streptomycin}, \mathrm{APX}=\mathrm{Ampiclox}, \mathrm{Z}=$ Zinacef (Cefuroxime), $\mathrm{R}=$ Rocephin (Ceftriaxone), $\mathrm{E}=$ Erythromycin 


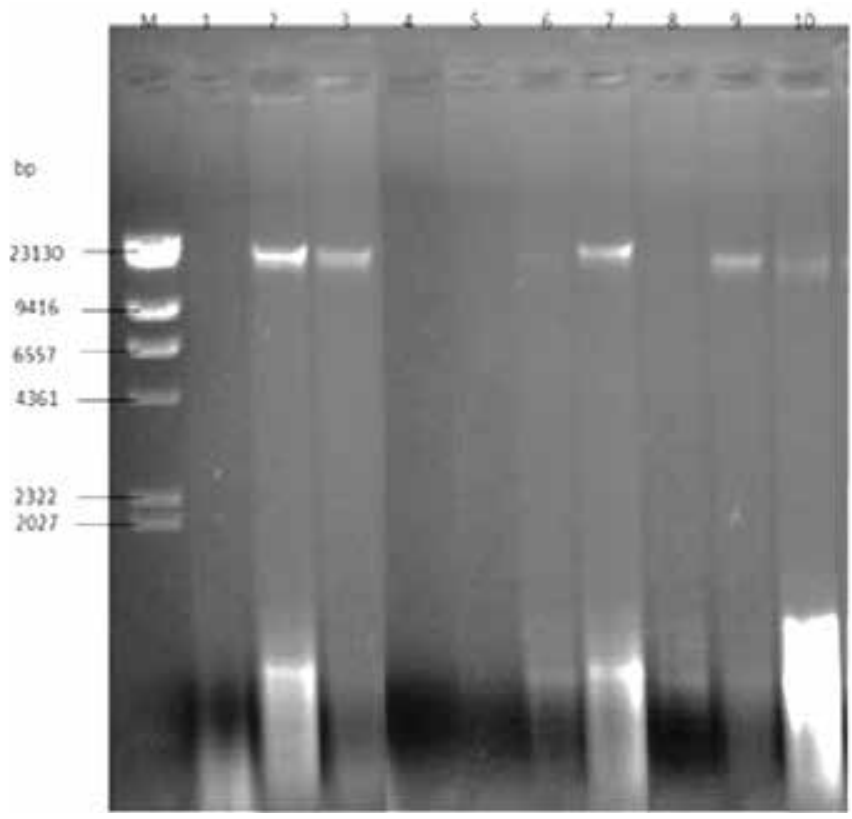

Fig. 1. 1\% Agarose gel of plasmid profiles of the bacterial isolates. Lane M, Molecular ladder, Lanes 1 - 10: 1, Pseudomonas aeruginosa; 2, Klebsiella sp.; 3, Bacillus subtilis; 4, Micrococcus luteus; 5, Streptococcus pyogenes; 6, Corynebacterium sp.; 7, Proteus vulgaris; 8, Staphylococcus epidermidis; 9, Staphylococcus aureus; 10, Escherichia coli.

ATM keypads were observed in this research to be contaminated with bacterial isolates. Similar results were noted by Bright et al. (2010), who accounted the presence of bacteria on frequently used fomites (Table 1). The prevalence of the bacterial isolates was coherent with that of Rutala et al. (2006) who isolated similar bacterial isolates from computer keyboards. Staphylococcus epidermidis followed by Staphylococcus aureus showed the utmost prevalence amongst other isolates. This observation is in agreement with that of Anastasiades et al. (2009) and Anderson and Palombo (2009), who showed that Staphylococcus aureus were more predominant on PC keypad and mouse. S. aureus is the chief constituent of the conventional flora of the skin and nostril, which undoubtedly clarify its elevated prevalence as contaminant, and can be effortlessly be emancipated by numerous human activities, like sneezing, speaking and contact with damp skin (Itah and Ben, 2004). The outcome of this study showed that the exteriors of routinely touched with hands harboured high bacteria load. One could definitely accept this to be true as most cross-contamination happens through this route. This is also in line with Anderson et al. (2008), who documented that students and other members of the public are not always the most diligent of hand-washers (Table II).
All the bacterial isolates exhibited varying resistance to the antibiotics used. Resistance of bacterial isolates to frequently used antibiotics has been observed in erstwhile studies, posing a public health difficulty (Khan and Malik, 2011; Tagoe and Kumi-Ansah, 2010). Manifestation of resistance in pathogens may drive down the efficacy of formerly suitable antibiotics (Toroglu and Dincer, 2008). The resistance of bacteria to antibiotic commonly in use is an increasing problem in many parts of the world and especially developing world (Giannini et al., 2009). Resistance of bacteria to Ampicillin, Amoxicillin, Chloramphenicol, Streptomycin, Trimethoprim-Sulfonamides and Gentamycin have been documented in different parts of Africa (Kimang'a, 2012) (Table III).

All the isolates from this research were multidrug resistant. Similar result had been stated by Nworie et al. (2012), who sequestered similar multi-drug resistant pathogens from door handgrip/handlebar in public lavatory and remarked that they serve as probable health hazards to an ever-increasing populace. There is also a great worry for the sequestration of multidrug-resistant Pseudomonas aeruginosa and Klebsiella sp., in this current study. Results showed Pseudomonas aeruginosa and Klebsiella sp. isolates to be resilient to Penicillins, Aminopenicillins, Aminoglycosides, Chloramphenicol and Trimethoprim/Sulfonamides groups of antibiotics, thus suggesting a threat to public health. Supporting findings had been reported by research works carried out by Rusin et al. (2002) and Nworie et al. (2012) who were of the opinion that such isolates portend health menace to the populace. Escherichia coli showing 100\% resistance (MAR index $=1$ ) to all 8 groups (Penicillins, Aminopenicillins, Cephalosporins, Aminoglycosides, Macrolides, Chloramphenicol, Fluoroquinolones and Trimethoprim/Sulfonamides) of antibiotics used in this study is quite worrisome. This agrees with the work of Issmat et al. (2007) who isolated multidrug-resistant bacteria from open shared interfaces. The results from this current research indicated that this public health menace still exist in our place of work/daily schedules. The high level of multidrug resistance (MAR index > 0.2) among the bacterial isolates makes the ATMs a source of community acquired infections. Elevated level of multi-antibiotic resilience demonstrated by Staphylococcus aureus and Staphylococcus epidermidis highlights the possibility of being potential etiological agents in pyrogenic infections. Staphylococcus aureus and Staphylococcus epidermidis have been associated in diverse infections, including septicaemia (Komolade and Adegoke, 2008). This outcome complies with the result of the investigation done by Tagoe and Kumi-Ansah (2010) where Staphylococcus aureus showed 83\% resistance to ampicillin and penicillin (Table IV). 
Curing of plasmid brought about a drop in the percentage resistance to Streptomycin, Gentamycin, Ampiclox and Chloramphenicol. The percentage drop in resilience to a handful of these antibiotics attributable to curing of plasmids suggests that a few of the resistance of the isolated bacteria to these antibiotics were plasmid-mediated. Plasmids have been accounted to bestow antibiotic resistance in a range of bacterial species (Normark and Normark, 2002). The plasmid profile of bacterial isolates depicts that resistance plasmids were detected in some of the bacterial isolates, with $E$. coli having an MAR index of $1(100 \%$ resistance to all the antibiotics) before curing and loss of plasmids to $80 \%$ resistance after curing. However, observation of the absence of plasmid in some of the isolates such as, Micrococcus luteus, suggests that the resistance may not be plasmid-mediated. This surveillance suggests that the prevalence of resistance in some of the isolates could be credited to other means (Chromosomal DNA resistance / inherent resistance), other than being plasmid-mediated. It has been investigated that antibiotic proneness of bacterial isolates is not unchanging but dynamic and fluctuates with time and location; this consequently stress the necessity for periodic assessment of conventional bacterial pathogens for their antibiotic susceptibility profiles in discrete communities (Rahman et al., 2007) (Table V and Fig. 1).

\section{Conclusion}

The presence of multidrug resistant bacteria on the keypads of Automated Teller Machines poses a great risk directly on the health of the individuals accessing this device and also indirectly on the health of the public at large, as the hands are highly effective vehicle for the transmission of clinically important pathogens. Members of the public take hygiene with levity, touching different dirty things with their bare hands, and then inoculating bacteria on the ATM keypads while pressing it. The findings of the study confirm the presence of potentially pathogenic multi-drug resistant bacteria on the keypads of the ATM and people should maintain good hand-washing routine. The use of hand-sanitizers which are portable and easy to use, for eliminating these organisms is recommended to avoid cross-contamination of microbes.

\section{Acknowledgement}

The authors would like to thank the Department of Microbiology, Faculty of Life Sciences, University of Benin, Benin City, Edo State, Nigeria, for providing materials for this work.

\section{References}

Akortha EE and Filgona J (2009), Transfer of gentamicin resistance genes among Enterobacteriaceae isolated from the outpatients with urinary tract infections attending 3 hospitals in Mubi, Adamawa State, Sci. Res. Ess. 4: 745 - 752.

Anastasiades P, Pratt TL, Rousseau LH, Steinberg WH and Joubert G (2009), Staphylococcus aureus on computer mice and keyboards in Intensive Care Units of The Universitas Academic Hospital, Bloemfontein and ICU staff's Knowledge of its hazards and cleaning practices, South Africa J. Epidem. Infect. 24: 22 - 26. DOI:10.1080/10158782.2009.11441345

Anderson G and Palombo EA (2009), Microbial contamination of computer keyboards in a University setting, Amer. J. Infect. Cont. 37: 507 - 509. DOI:10.1016/ j.ajic.2008.10.032

Anderson JL, Warren CA, Perez E, Louis R I, Phillips S, Wheeler J, Cole M and Misra R (2008), Gender and ethnic differences in hand hygiene practices among college students, Amer. J. Infect. Cont. 36: $361-368$. DOI:10.1016/j.ajic.2007.09.007

Bauer AW, Kirby WMM, Sherris JC and Turck M (1966), Antibiotic susceptibility testing by a standardized single disk method, Amer. J. Clin. Path. 45: 493 - 496.

Bieser AM, Thomann Y and Tiller JC (2011), Contact-active antimicrobial and potentially self-polishing coatings based on Cellulose, Macromol. Biosci. 11: 111 - 121. DOI:10.1002/mabi.201000306

Breed RS, Murray EGD and Smith WR (1975), Bergeys Manual of Systematic Bacteriology, $7^{\text {th }}$ Ed. Williams and Wikins, Baltimore, MD, USA.

Bright KR, Boone SA and Gerba CP (2010), Occurrence of bacteria and viruses on the Elementary Classroom surfaces and the potential role of Elementary Classroom hygiene in the spread of infectious diseases, J. Sch. Nurs. 26(1): 33 - 41. DOI:10.1177/1059840509354383

CLSI (Clinical and Laboratory Standard Institute) (2013), Performance Standards for Antimicrobial Disk Susceptibility Tests, Approved Standard (M100-S23), 33(1): 1 - 199.

Giannini MA, Nance D and McCullers JA (2009), Are toilet seats a vector for transmission of methicillin-resistant Staphylococcus aureus?, Amer. J. Infect. Cont. 37: 505 - 506. DOI:10.1016/j.ajic.2008.11.005 
Hamouda T and Baker J (2000), Antimicrobial mechanism of action of surfactant lipid preparations in enteric Gram-negative Bacilli, $J$. App. Microbiol. 89: 397 - 403.

Holt JG, Krieg NR, Sneath PHA, Staley JT and Williams ST (1994), Bergey's manual of determinative bacteriology, $9^{\text {th }}$ Ed., William and Wilkins, Baltimore, MA, USA.

Issmat IK, Von S and Malak AE (2007), Public computer surfaces are reservoir for methicillin-resistant Staphylococci, The Inter. Soc. Microb. Ecol. J. 1: 265 - 268. DOI:10.1038/ismej.2007.36

Itah AY and Ben AE (2004), Incidence of enteric bacteria and Staphylococcus aureus in day care centres in Akwa Ibom, State, Nigeria, Southeast Asian J. Trop. Med. Pub. Health 35(1): 202 - 209.

Khan RMK and Malik A (2011), Antibiotic resistance and detection of $\beta$-lactamase in bacterial strains of Staphylococci and Escherichia coli isolated from foodstuffs, World J. Microbiol. Biotech. 17: 863 - 868.

Kimang'a AN (2012), Review article a situational analysis of antimicrobial drug resistance in Africa: Are we losing the battle?, Ethiop. J. Health Sci. 22: 135 - 140.

Komolade AO and Adegoke AA (2008), Incidence of bacterial septicemia in Ile-Ife, Nigeria, Malaysian $J$. Microbiol. 4(2): 51 - 61.

Maillard JY (2002), Bacterial target sites for biocide action, J. Appl. Microbiol. 92: 16 - 27.

Maillard JY (2005), Antimicrobial biocides in the healthcare environment: Efficacy, Usage, Policies, and Perceived Problems, Therap. Clin. Risk Manag. 1: 307 - 320.

Mathai E, Allegranzi B, Kilpatrick C and Pittet, D (2010). Prevention and control of health care-associated infections through improved hand hygiene, Indian J. Med. Microbiol. 28: $100 \quad$ - 106. DOI: $10.4103 / 0255-0857.62483$

NCCLS (National Committee for Clinical Laboratory Standards) (2000), Methods for dilution antimicrobial susceptibility tests for bacteria that grow aerobically, $5^{\text {th }}$ Ed., NCCLS document M7-A5, American Society for Microbiology, Washington DC.

NCCLS (National Committee for Clinical Laboratory Standards) (2005), Performance Standards for antimicrobial susceptibility testing, $15^{\text {th }}$ informational supplement (M100-S15), National Committee for Clinical Laboratory Standards, Wayne PA.

Normark BH and Normark S (2002), Evolution and spread of antibiotic resistance. J. Int.l Med. 252: 91 - 106.
Nworie A, Ayeni JA, Eze UA and Azi SO (2012), Bacterial contamination of door handle/knobs in selected public conveniences in Abuja Metropolis, Nigeria: A threat to public health, Continent. J. Med. Res. 6(1): 7 - 11 .

Rahman M, Rahman B, Rahman T, Khan FR and Hosen MJ (2007), Patterns of antimicrobial resistance and plasmid profiles of Escherichia coli isolates obtained from calf, cattle and diarrheic children in Mymensingh-Bangladesh, J. Amer. Sci. 3: 74 - 84.

Rusin P, Maxwell S and Gerba C (2002), Comparative surface-to-hand and fingertip-to-mouth transfer efficiency of Gram-positive bacteria, Gram-negative bacteria, and phage, J. App. Microbiol. 93: 585 - 592.

Rutala WA, White MS, Gergen MF and Weber DJ (2006), Bacterial contamination of keyboards: efficacy and functional impact of disinfectants, Infect. Cont. Hos. Epidemiol. 27: 372 - 377. DOI:10.1086/503340

Saroja V, Kamatchiammal S, Brinda K and Anbazhagi S (2013), Enumeration and characterisation of coliforms from Automated Teller Machine (ATM) centers in urban areas, J. Mod. Biotech. 2(1): $14-22$.

Sharma M and Anand SK (2002), Biofilms evaluation as an essential component of HACCP for food/dairy processing industry - a case, Food Control 13: 469 477. DOI:10.1016/s0956-7135(01)00068-8

Short AD, Kennedy LJ, Barnes A, Fretwell N, Jones C, Thomson W and Ollier WE (2007), Hardy Weinberg expectations in canine breeds: implications for genetic studies, J. Heredity 98: 445 - 451. DOI:10. 1093/jhered/esm020

Steffen E, Edith F, Arne S, Jurgen G, Stefanie B and Martin E (2008), Microbial contamination of computer user interfaces (keyboard, mouse) in a tertiary care centre under conditions of practice, Hyg. Med. 33(12): 504 507.

Tagoe DNA and Kumi-Ansah F (2010), Computer keyboard and mouse: Potential sources of disease transmission and infections, Inter. J. Pub. Health. 1: 2 - 9

Toroglu S and Dincer S (2008), Elimination and conjugal transfer of their resistance in isolated Gram negative bacteria from Aksu River (Kahramanmaras-Turkey), Adv. in Environ. Biol. 2: 124 - 131.

Zhou C, Yujan Y and Ambrose YJ (1990), TENS- Mini-prep for isolation of plasmids, J. Clin. Microbiol. 34: 234 240 . 